\title{
Competition between Insurers with Superior Information*
}

\author{
Bertrand Villeneuve ${ }^{\dagger}$ \\ Université de Toulouse I (CEA, IDEI and LEERNA)
}

December 2002.

\begin{abstract}
We analyze markets where insurers are better informed about risk than consumers. We show that even competitive markets may result in insufficient information revelation and inefficient insurance coverage. This explains why certain risky consumers remain unsinsured and why certain market segments are persistently profitable. We also show robustness to competition in menus or mechanisms. Our analysis of the "contrary of adverse selection" (competition between principals with common value and exclusivity) is suitable for other markets (lawyers, doctors, mechanics, etc.).
\end{abstract}

JEL: D43, D82, G22.

Keywords: informed principals; common value; competition in mechanisms; insurance markets; adverse selection.

${ }^{*}$ This paper has circulated under several titles. Discussions with Pierre-André Chiappori, Jacques Crémer, Roberta Dessi, Georges Dionne, Nathalie Fombaron, Guido Friebel, Joe Harrington, Charles Kahn, Jean-Jacques Laffont, Lambros Pechlivanos, JeanCharles Rochet, Bernard Salanié, Jean Tirole, Désiré Vencatachellum, Michael Warlters and Michael Whinston helped me improve the exposition. I am very grateful to Klaus Schmidt and the referees for their extremely useful suggestions. All errors are mine.

${ }^{\dagger}$ Address: IDEI, University de Toulouse I, Place Anatole France, 31042 Toulouse cedex, France. E-mail: bertrand.villeneuve@cict.fr. 


\section{Introduction}

Experts like lawyers, doctors, mechanics or coaches, combine knowledge of the state of their art and of its application to the particularities of their customers. Expert's skill in this kind of relationship has more weight on the right decision than hidden information the consumer may possess. Above all, many experts are not only selling information but also the derived commodities or services, which increases the conflict of interest they face.

This article considers competition between informed principals (experts) with common value and exclusivity. We show the reasons why competition does not ensure perfect revelation of information to consumers, why, even if market interactions reveal information, the allocation may remain inefficient, and finally why part of the surplus may remain in the hands of some firms.

We put a general argument in insurance terms, but similar features would emerge in other markets where the experts advise and sell. Other applications only require that the cost to the provider and the preferences of the customer both depend on the same hidden parameters (common value) and that the customer has access to one provider only at the same time (exclusivity).

Adverse selection is recognized as a serious threat to insurance markets, but empirical evidence of its existence is scarce (see Puelz and Snow, 1994). The very success of insurers' classification methods certainly explains to a large extent the paradox that adverse selection is both feared and hardly visible. This is the point made by Chiappori and Salanié (2000). Though their results cannot be generalized to the whole insurance industry, ${ }^{1}$ we have to consider seriously the possibility that the risk predictors insurers use for fear of adverse selection might be more precise than customers'.

This view could be dismissed as unrealistic in markets where individual factors are essential, such as in life and health insurance, but the risks covered by the insurance industry are extremely varied. Every example is controversial, but companies specialized in the insurance of activities where damages are rarely experienced can reasonably be expected to better anticipate the possible financial consequences of an accident. For instance, in liability insurance, up-to-date knowledge of the jurisprudence is required and most clients are not really aware of the compensations they could be forced to pay.

This paper provides a counterpoint to Rothschild and Stiglitz (1976). In this model, insurers are assumed to know precisely the structure of their clientele in terms of risks, tastes and beliefs, so as to be able to establish classes of clients and to practise price discrimination. The right given to

\footnotetext{
${ }^{1}$ Cohen (2001) shows evidence that adverse selection in automobile insurance markets can be observed.
} 
insurers to discriminate is based on the idea that every individual is a different market. ${ }^{2}$ (This is discussed in the presentation of the model and in Subsection 4.5.) No informational difference between insurers is assumed. The policyholder does not know whether he is a low or a high risk. Insurers observe, before contracting, the type of the applicant; they make personal offers simultaneously; the individual is able to interpret offers as informative signals, and remains free to accept one or to reject them all. A Bayesian equilibrium concept guarantees a consistent understanding of observed actions on the part of the consumer. ${ }^{3}$

The common view is that classification poses problems of distributive justice - higher risks pay more - not of efficiency. ${ }^{4}$ The intuition is that competition enables the consumer to exploit the conflicting interests of the various providers to get the best information, the most appropriate quality, and the lowest price. Auction theory supports this optimism: in the simple situation where bidding firms are identical in terms of technology and information (so that first- and second- prices will be identical), an auction ensures efficiency and allows the consumer to overcome his inferior information and to claim all the surplus.

Further, the common view is that competition should result in full revelation of available information (the consumer's type) and first-best efficiency (full insurance at an actuarially fair rate). This article proves that this is not the case: types may be pooled, the high risk consumers may remain without insurance or obtain partial coverage, and profits are not always zero. ${ }^{5}$

The intuition for inefficient though fully informative equilibria is as follows. Consider the case where low risk individuals are fully and fairly insured while high risk individuals are not insured at all. Obviously, insurance companies would like to offer full insurance to high risk individuals since this would be profitable and even Pareto improving. One the other hand, individuals can only infer their types from the offers they receive. Any "claim" by an insurer that the customer is a high risk poses a credibility problem: an insurer would always like to make a low risk think he is a high risk (this

\footnotetext{
${ }^{2}$ Even if data on sex, race, religion, etc., cannot be used, or even recorded, many other items of information can.

${ }^{3}$ See Villeneuve (2000) (informed insurance monopoly) for comparisons with Stiglitz' (1977) (insurance monopoly with adverse selection).

${ }^{4}$ Classification, when it goes too far, has a dark side: mutualization possibilities that were used previously are definitively thrown away, thus generating greater inequality in the economy. See Crocker and Snow (2000) for a recent survey.

${ }^{5}$ Fagart (1996) explored a similar model with compulsory insurance. This forced participation of the agent implies that information is systematically revealed and that profits are zero. We show that non-participation threats explain important features of the equilibrium.
} 
increases his willingness-to-pay for insurance), and the consequence is that such statements are not automatically believed. Indeed, assume the consumer believes that he is a low risk with high probability whenever he is offered anything other than no-insurance by one of the insurers. If he is a low risk, fine, competition works well and he gets the first-best. If he is a high risk, all insurers "offer" him no insurance. Why is this an equilibrium? The consumer's interpretation rule plays a key role: if an individual insurer deviates and offers him, say, the full insurance contract a high risk deserves, the individual thinks he is a low risk. If he refuses the offer because it is too expensive, deviations are discouraged, and the high risk customer finishes up uninsured. ${ }^{6}$ The key point now is whether individuals believing they have low risk are reluctant to accept contracts suitable for high risk customers. This depends on the "proximity" between extreme types: if types are very different, then contracts that are profitable on high risk types may never be acceptable for customers believing they have a low risk with a probability sufficiently close to one.

Pooling equilibria (in which no information is revealed) follow a slightly different logic. Take a situation where $N$ insurers offer the same contract $C$ to all types. Obviously, this contract must be acceptable for the individual who has not learned his type, and profitable on both types. When is this stable? A priori, an individual insurer is tempted to deviate when a low risk shows up by offering him a better contract: instead of sharing the customer (i.e. earning a large profit with probability $1 / N$ only), it would earn a lower profit with probability one. Now assume that the consumer concludes that he is a low risk with probability one whenever he observes something else than $C$ being offered. With these optimistic beliefs, deviations that are accepted by the customer with probability one are not necessarily sufficiently profitable compared to $C$, and, consequently, sufficiently profitable deviations are not accepted. In any case, the allocation is stable. A pooling equilibrium does not always exist, but, surprisingly enough, it can exist with an infinite number of insurers. Two conditions have to be met: the low risk must be low enough for willingness-to-pay for insurance to be sufficiently low if the consumer is optimistic, and high risk individuals have to be sufficiently more likely for the willingness-to-pay for insurance of the uninformed consumer to be sufficiently high.

Related literature Our work is technically linked with the literature on informed principals and on credence goods. Leaving the initiative to the in-

\footnotetext{
${ }^{6}$ The credibility issue is so strong that these beliefs are compatible with strong refinements (such as Cho-Kreps).
} 
formed agent is also the core hypothesis of Maskin and Tirole (1992). The essential difference is that here, the uninformed party confronts several (rather than one) informed parties. ${ }^{7}$ The strategy space used in Maskin and Tirole (1992) is also worth mentioning: the informed party proposes a "mechanism" (a game form), which improves the efficiency of the equilibria. This paper shows that, with multiple principals, this effect is extremely limited (Subsection 4.3). In the literature on credence goods (e.g. Wolinsky, 1995, or Emons, 1997), the hidden parameter is a private value, i.e. it only affects the consumer's willingness-to-pay, not provision cost. Our work can be seen as the common value version of this research area. In Hertzendorf and Overgaard (2001), two producers compete in prices, a common feature with our model being that the pair of prices is treated globally as a signal about producers' qualities, which are different. In this paper, producers are identical, and we show that it is not a sufficient condition for the market to work properly.

\section{The Model}

A consumer with wealth $W$ incurs the risk of losing a monetary amount $d$. Initially, the loss probability (the type) is known to the individual and the insurers only up to its distribution in the population. We assume that the consumer is a high-risk type (loss probability $p_{H}$ ) with probability $\lambda_{H}>0$ and a low-risk type (loss probability $p_{L}$ with $0 \leq p_{L}<p_{H}$ ) with probability $\lambda_{L}=1-\lambda_{H}>0 .{ }^{8}$ The average loss probability is denoted by $p_{A} \equiv \lambda_{H} p_{H}+$ $\lambda_{L} p_{L}$. The concave VNM utility function of the agent is denoted by $u$, and the insurers are assumed to be risk-neutral profit maximizers.

The insurers are indexed by a set $I=\{1,2, \ldots, N\}, N \geq 2$. The time structure of the game is as follows:

1. The consumer's type $(H$ or $L)$ is randomly selected.

2. All insurers observe the type, but the consumer remains uninformed.

3. The insurers simultaneously offer contracts.

4. The consumer observes the offers, updates his priors, and selects exclusively the preferred offer or the reservation contract (no insurance).

\footnotetext{
${ }^{7}$ An unessential difference is that, in their application to insurance, the informed party is the policyholder. We have in common that there is one policyholder and several insurers.

${ }^{8}$ Extension to distributions with large supports does not substantially change our main point. Extension to more than two types, however, necessitates a particular treatment given in Subsection 4.3.
} 
5. The loss occurs or not and the accepted contract is implemented.

Let $\Omega$ be the contract space (specified later), and let us denote by $C^{i} \in \Omega$ insurer $i$ 's offer. The "interpretation mapping" (or simply the beliefs) of the policyholder maps the set of vectors of offers $\Omega^{N}$ into $\Delta(\{H, L\})$, the set of probability distributions over types. In fact we can sum up beliefs by $\widetilde{p}\left(C^{\bullet}\right) \in\left[p_{L}, p_{H}\right]$ (the perceived risk probability) where $C^{\bullet} \equiv\left(C^{i}\right)_{i \in I}$ is a vector of offers. The consumer's weak preference order over $\Omega$ that a certain belief $\widetilde{p}$ implies will be denoted by $\succeq_{\tilde{p}}$. Beliefs are endogenous and known to insurers in equilibrium (they anticipate correctly the consequences of their actions on beliefs).

An offer will be a contract actually proposed by an insurer, whereas the allocation will be the (set of) contract(s) actually adopted by the consumer in equilibrium. A contract is (strictly) acceptable for beliefs $\widetilde{p}$ if it is (strictly) preferred to no insurance given the beliefs. Similarly, a contract is (strictly) profitable on type $K(K=H, L)$ if an insurer earns (strictly) positive profit on this contract when it is accepted by a type- $K$ consumer.

The reservation contract $C^{0}$ (here the right to remain uninsured) plays a fundamental role, as we shall see. The decision of the individual at stage 4 will respect the following rules: given the revised priors, (a) any contract which is strictly dominated is never taken; (b) if there are two or more optimal contracts, the individual chooses randomly and symmetrically among optimal offers differing from no-insurance. In particular, the consumer accepts any offer with probability $1 / N$ whenever all insurers make optimal offers. Our tie-breaking rule ensures that the individual's strategy is uniquely determined by his beliefs.

We focus our analysis on the Perfect Bayesian Equilibria (PBE) in pure strategies. ${ }^{9}$

Definition 1 A PBE is: (a) a set of offers $\left(C_{H}^{\bullet *}, C_{L}^{\bullet *}\right)$ where $C_{K}^{i *}$ is the offer that insurer $i$ makes to a consumer of type $K$ and (b) a belief mapping $\widetilde{p}$ such that:

1. Actions are sequentially optimal: for any type $K$, for any insurer $i, C_{K}^{i *}$ maximizes the profit of insurer $i$ in $\Omega$ given (i) $C_{K}^{-i *}$ (the actions of the other insurers) and (ii) $\widetilde{p}\left(C_{K}^{-i *}, \cdot\right)$ (its impact on beliefs given the others' actions).

2. Beliefs are consistent with Bayes rule: if $\left(C_{H}^{\bullet *}\right)=\left(C_{L}^{\bullet *}\right)$ then $\widetilde{p}\left(C_{H}^{\bullet *}\right)=$ $\widetilde{p}\left(C_{L}^{\bullet *}\right)=p_{A} ;$ else $\widetilde{p}\left(C_{H}^{\bullet *}\right)=p_{H}$ and $\widetilde{p}\left(C_{L}^{\bullet *}\right)=p_{L}$; they are not restricted elsewhere.

\footnotetext{
${ }^{9}$ Equilibria in pure strategies abound, therefore we leave equilibria in mixed strategies (sometimes called semi-separating equilibria in signalling games) aside to avoid confusion.
} 
Individual Markets In this analysis, each customer is an "individual market". Strictly speaking, this means that there is a different equilibrium for every customer, consequently, two individuals with the same initial characteristics (same priors, same risk) may end up with different allocations. This assumption is not problematic for very heterogenous markets, like insurance for factories or buildings, or for groups that are hard to target. Indeed, when the technology to reach the members of a group is lacking, public offers to larger audiences tend to mix members of the group and consumers that insurers would prefer to treat differently. This may lead insurers to make individual rather than general offers. Once the functioning of individual markets is well understood, it becomes easier to discuss the notion of public offers and their consequences. This is done in Subsection 4.5.

Strategy Spaces Three different insurer strategy spaces $\Omega$ are investigated: $\Omega_{0}$ for full insurance contracts, $\Omega_{1}$ for insurance contracts in the ordinary sense, and $\Omega_{2}$ for the mechanisms. $C^{i}$ in $\Omega_{0}$ or $\Omega_{1}$ specifies consumptions $W_{A}^{i}$ in case of loss and $W_{N}^{i}$ in case of no loss $\left(C^{i} \equiv\left(W_{N}^{i}, W_{A}^{i}\right)\right.$; note in particular that $\left.C^{0} \equiv(W, W-d)\right) .{ }^{10}$ We only consider "positive" insurance contracts for which $W_{A} \geq W-d$ and $W_{N} \leq W^{11}$ The full set of insurance contracts is denoted by $\Omega_{1}$; in particular over-insurance $\left(W_{A}>W_{N}\right)$ or under-insurance $\left(W_{A}<W_{N}\right)$ contracts are not excluded. $\Omega_{0}$ will denote the subset of full insurance (i.e. constant consumption, or $W_{A}^{i}=W_{N}^{i}$ ) contracts; two contracts in this set only differ by the premia. ${ }^{12}$ Finally, $\Omega_{2}$ is the set of mechanisms (or game-forms) as in Maskin and Tirole (1992).

We use the following notations with $K=H, L$ or $A$ (for the uninformed average):

$\pi_{K}(C)$ : expected profit made on type $K$ if $C=\left(W_{A}, W_{N}\right)$ is taken:

$$
\pi_{K}(C) \equiv\left(1-p_{K}\right)\left(W-W_{N}\right)+p_{K}\left(W-d-W_{A}\right)
$$

$C_{K}^{\mathrm{AF}}$ : the actuarially fair (= zero profit) full-insurance contract of type $K$.

$C_{K}^{\max }$ : the contract extracting maximal profit under symmetric information (see equation 2) on type $K$.

\footnotetext{
${ }^{10}$ Defining contracts in terms of premium (paid in advance before the loss is known) and indemnity (paid in case of loss) is equivalent.

${ }^{11}$ We can always find sustainable off-equilibrium beliefs such that negative insurance cannot be offered as a deviation: it suffices to see that for each type ( $H, L$ or average), a contract that is profitable is not acceptable and vice versa.

${ }^{12}$ Notice that though $C^{0} \notin \Omega_{0}$, no insurance remains the reservation contract.
} 
The notions defined above are represented in the plane $\left(W_{N}, W_{A}\right)$ (see Figure 1). " $U_{K}$ " indicates a type- $K$ indifference curve; " $\pi_{K}$ " indicates a type- $K$ isoprofit straight line.

Insert Figure 1 Here

Section 3 solves the model for full insurance contracts $\left(\Omega_{0}\right)$ and presents the main intuitions and practical implications of our analysis. Section 4 shows the resistance of inefficiencies when insurers' strategies are extended to more general contracts $\left(\Omega_{1}\right)$ or mechanisms $\left(\Omega_{2}\right)$. We also offer an extension to more than two types of individuals, and discus the effects of refining beliefs. We discuss the impact of public offers and of mandatory insurance. The conclusion focuses on insurance regulation and on the testability of the model. Proofs are relegated to the Appendix.

\section{Price Competition}

Constraining offers to provide full insurance simplifies the exposition, and above all, sets the best conditions for competition to work efficiently: offers differ only with respect to prices and comparisons are not type-dependent. However, we show that competition does not lead necessarily to equilibria with discrimination (information is not necessarily revealed) and equilibrium profits are not systematically zero (the Bertrand logic is not fully effective).

In this section, a full insurance contract will be simply denoted by the contribution $c$ that is charged to the consumer, the indemnity being equal to $d$. Five contracts play a particular role: $C_{H}^{\mathrm{AF}}, C_{L}^{\mathrm{AF}}, C_{H}^{\max }, C_{L}^{\max }$ and $C_{A}^{\max }$ : the minimum price that insurers charge is $c_{H}^{\mathrm{AF}}=p_{H} d$ for an $H$ and $c_{L}^{\mathrm{AF}}=p_{L} d$ for an $L$; conversely, a consumer aware of his type $K(=H, L$ or $A$ if he is not informed at all) is willing to pay a maximum of $c_{K}^{\max }$, where

$$
u\left(W-c_{K}^{\max }\right)=\left(1-p_{K}\right) u(W)+p_{K} u(W-d), K=H, L, A
$$

Note that $c_{H}^{\mathrm{AF}}<c_{H}^{\max } ; c_{L}^{\mathrm{AF}}<c_{L}^{\max } ; c_{L}^{\mathrm{AF}}<c_{H}^{\mathrm{AF}} ; c_{L}^{\max }<c_{A}^{\max }<c_{H}^{\max }$.

\subsection{Discrimination}

We study first equilibria where different types are treated differently.

Proposition 1 A separating equilibrium always exists. In any equilibrium:

1. The low risk is charged the actuarially fair price $c_{L}^{A F}=p_{L} d$, and at least two insurers charge this price. 
2. If $c_{L}^{\max }>c_{H}^{A F}$, the high risk is charged the actuarially fair price $c_{H}^{A F}=$ $p_{H} d$, and at least two insurers charge this price.

3. If $c_{L}^{\max } \leq c_{H}^{A F}$, no insurance $C^{0}$ and any price in $\left[c_{H}^{A F}, c_{H}^{\max }\right]$ are equilibrium allocations for the high risk. For no-insurance equilibria, all prices offered are above $c_{L}^{\max }$. In the other equilibria, the price paid is the lowest price among those offered.

The first important fact is that the actuarially fair full insurance allocation is always an equilibrium: competition can work. However, other types of equilibria appear, notably those involving the high risk being either exploited (unfair premium), or not insured.

The difference between the maximum an $L$ would accept to pay $\left(c_{L}^{\max }\right)$ and the minimum an $H$ should be charged $\left(c_{H}^{\mathrm{AF}}\right)$ is critical, whereas the number of insurers is irrelevant. Moreover the distribution of types $\left(\lambda_{H}\right.$ and $\left.\lambda_{L}\right)$ does not matter since the allocation is separating. The difference between types determines the possible regimes. For a fixed $p_{H}, c_{L}^{\max }>c_{H}^{\mathrm{AF}}$ if $p_{L}$ is close to $p_{H}$, whereas $c_{L}^{\max }<c_{H}^{\mathrm{AF}}$ if $p_{L}$ is sufficiently small. The threshold between these two cases is the solution $p_{L}$ of $c_{L}^{\max }=p_{H} d$ in which the LHS is an increasing function of $p_{L}$. A similar argument shows that, for fixed loss probabilities $p_{H}$ and $p_{L}$, we have profitable equilibria if risk aversion is high, and only actuarially fair contracts if risk aversion is low. Whenever it is possible that the consumer is not at risk $\left(p_{L}=0\right.$ and $\left.\lambda_{L}>0\right)$, then the contract that the high risk gets may be anything from an actuarially fair contract to a maximal price contract, plus no-insurance.

Competition is harsh and efficient when types are approximately alike (in a subjective sense since risk aversion matters): even optimistic individuals are not reluctant to accept contracts suited for the high risk. As a consequence, insurers are always encouraged to lower prices to attract consumers. When types are sufficiently dissimilar, inefficient or unfair allocations can be stabilized if the individual is overly optimistic off the equilibrium path. Indeed, if the consumer happens to be a high risk, cutting high prices may be misinterpreted as a signal that he is a low risk, which leads him to reject the better offer and remain uninsured. Competition is so strongly hindered that a firm may capture all the rent without being seriously threatened by its competitors.

\subsection{Uniform Pricing}

In a pooling equilibrium, each insurer $i$ charges the same price $c^{i *}$ for all types, but two different insurers could charge different prices $\left(c^{i *} \neq c^{j *}\right.$ if 
$i \neq j$ ). We show that all offers are identical in a pooling equilibriumwe denote the only offer by $c^{*}-$, and $c^{*}$ is different from and preferred to no-insurance. Indeed, the allocation is the same for all types, since they receive the same offers and make the same choice. Given that whatever his subsequent beliefs, a consumer would accept any offer which is profitable and acceptable for the most demanding belief $p_{L}$, the allocation differs from noinsurance since competition ensures that type- $L$ agents are not left without insurance. Indeed, an insurer offering something other than $c^{*}$ would only have to offer a slightly profitable contract $\left(c>c_{L}^{\mathrm{AF}}\right)$ when the consumer is an $L$ to be better-off. This would break the equilibrium.

Whether or not $c^{*}$ is sustainable is determined by the two following conditions:

(a) $c^{*}$ must be profitable on all types, and acceptable for the average type (the consumer keeps his priors). This implies $p_{H} d \leq c^{*} \leq c_{A}^{\max }$. (b) Any offer smaller than $c_{L}^{\max }$ is automatically acceptable, therefore each insurer must offer $c^{*}$ (and earn the profit with probability $1 / N$ ) to attracting a type- $K$ consumer for sure with $c_{L}^{\max }$ (or less). ${ }^{13}$ This implies $\frac{c^{*}-p_{K} d}{N} \geq c_{L}^{\max }-p_{K} d, K=$ $H, L$.

An example of appropriate beliefs is the following: for vectors of offers differing from $\left(c^{*}, c^{*}, \ldots, c^{*}\right)$, let the individual believe that he is a low risk. ${ }^{14}$ This optimistic attitude discourages deviations: out of the equilibrium, the individual's willingness-to-pay for insurance being always minimal, an insurer sees a deviation either as non-profitable, or, as non-acceptable given the beliefs.

We now analyze the dependency of existence on the following parameters: $p_{H}, p_{L}, \lambda_{H}$ (or, equivalently, $\lambda_{L}$ ), and $N$.

Proposition 2 Statements are given ceteris paribus.

1. The set of pooling allocations becomes larger as $\lambda_{H}$ increases.

2. If $p_{L}>0$, there is a finite upper bound on $N$ (dependent of $p_{L}$ ) consistent with pooling equilibria.

3. For any $N$, if $p_{L}$ and $\lambda_{L}$ are small enough, then pooling equilibria exist.

A pooling allocation becomes less profitable as the number of insurers increases (point 2). However, the benefit of deviating may be very low if $L$ is a very low risk (point 3): the possibility in the consumer's mind that he

\footnotetext{
${ }^{13}$ Remark that any offer higher than $c_{L}^{\max }$ can be discouraged by making the consumer believe he is a $L$ when he sees it.

${ }^{14}$ We show in Subsection 4.4 that such extreme rules are sufficient but not necessary.
} 
might be a very low probability type is sufficient for a pooling equilibrium to exist. In particular if $p_{L}=0$, if the proportion of low-risk consumers is low enough, then whatever $N$, there are pooling equilibria.

This result has considerable practical importance since, in most insurance markets, people with very low risk do exist; even if they are very few, the consumer may tend to identify himself with them, and non-discriminating strictly profitable offers have to be expected. Both types being profitable in a pooling equilibrium, the absence of discrimination indicates the lack of competitive pressure.

\section{Generalization}

Whenever offers differ from full insurance contracts, we observe two types of consequences. First, certain equilibria of price competition disappear because we have extended the possibilities of deviation. Second, new equilibria appear where the consumer receives something other than full insurance. We shall insist more on equilibria that subsist and on the reasons why they subsist. We limit ourselves here to giving a few indications of their appearance. ${ }^{15}$

\subsection{Competition in Contracts}

Separating Allocations We now distinguish three degrees of proximity between types:

D: Types are Distant if and only if no contract profitable on $H$ is acceptable for belief $p_{L}\left(\frac{p_{L}}{1-p_{L}} \frac{u^{\prime}(W-d)}{u^{\prime}(W)}<\frac{p_{H}}{1-p_{H}}\right.$ and $\left.C_{H}^{\mathrm{AF}} \prec_{p_{L}} C^{0}\right)$.

WR: Types are Weakly Related if and only if, at the actuarially fair price of the high risk, marginal insurance is acceptable for belief $p_{L}$, but not complete insurance $\left(\frac{p_{L}}{1-p_{L}} \frac{u^{\prime}(W-d)}{u^{\prime}(W)} \geq \frac{p_{H}}{1-p_{H}}\right.$ but $\left.C_{H}^{\mathrm{AF}} \prec_{p_{L}} C^{0}\right)$.

CR: Types are Closely Related when the actuarially fair full insurance of the high risk is acceptable for belief $p_{L}\left(\frac{p_{L}}{1-p_{L}} \frac{u^{\prime}(W-d)}{u^{\prime}(W)} \geq\right.$ $\frac{p_{H}}{1-p_{H}}$ and $\left.C_{H}^{\mathrm{AF}} \succeq_{p_{L}} C^{0}\right)$.

(Note that in $\Omega_{0}$-price competition- $\mathbf{D}$ and $\mathbf{W R}$ both correspond to $c_{L}^{\max }<c_{H}^{\mathrm{AF}}$.)

We have an ordered partition: given $p_{H}$, it is necessary and sufficient to make $p_{L}$ pass from 0 up to $p_{H}$ for passing from regime $\mathbf{D}$ to regime $\mathbf{W R}$

\footnotetext{
${ }^{15}$ Readers interested can request the former working paper.
} 
and then to regime $\mathbf{C R}$; conversely, given $p_{L}$, it is necessary and sufficient to make $p_{H}$ pass from 1 down to $p_{L}$ for passing through the three regimes. See Figure 2.

\section{Insert Figure 2 Here}

Actuarially-fair full insurance for all is always an equilibrium. In regime CR, beliefs do not matter very much and the allocation is relatively efficient: competition ensures that all types get an actuarially fair insurance. Regime D is particularly interesting: $H$ may get anything (full insurance, underinsurance or overinsurance) provided it is profitable and acceptable. Regime WR is an intermediary situation that is particular to competition in contracts: $H$ cannot get a slightly profitable contract because there are equally profitable contracts that are always acceptable. Consequently, profits on $H$ are either zero or above a minimum, depending on the parameters. In general, the quality of coverage may be suboptimal for both types because quality improvements (like cheaper contracts) are misinterpreted and discouraged.

Pooling Allocations A pooling equilibrium allocation of price competition is always an equilibrium allocation of contract competition. Indeed, when all cheaper full insurance contracts are either unattractive for some beliefs or less profitable to the firms, then this is also the case for less efficient contracts (underinsurance or overinsurance). In other terms, if we can't break a pooling with full insurance contracts, then there exist off-equilibrium beliefs such that no other contract breaks the equilibrium. The difference however is that there are other equilibria in which the contract offered is not full insurance: for the same reason that discourages cheaper offers, more efficient offers can be discouraged.

\subsection{Competition in Menus ${ }^{16}$}

Competition in mechanisms is hard to tackle directly. Nevertheless we build on the previous results to show why inefficiency persists. To understand the practical meaning of this game, one can see mechanisms in the following way: by offering a mechanism $\mu_{i}$, insurer $i$ pre-commits to a short list of allocations $\left\{C_{j}^{i}\right\}_{j \in J}$, where $J$ is a sufficient set of indices (two contracts with different indices may be identical); once offer $\mu_{i}$ is accepted, insurer $i$ can impose any of the allocations in $\left\{C_{j}^{i}\right\}_{j \in J}$ to the consumer.

\footnotetext{
${ }^{16}$ We thank Michael Whinston for suggesting that we examine this question.
} 
Contrarily to the previous strategy spaces, we are not able to propose here a clear-cut typology of the equilibria. Nevertheless, we find that if types are sufficiently dissimilar, profitable equilibria exist.

Proposition 3 Fix $p_{H}$ and $p_{L}$ and consider a symmetric separating equilibrium of contract competition in which $L$ receives $C_{L}^{A F}$. We denote by $C_{H}$ the contract received by $H$ (no insurance, a profitable contract or a fair contract, depending on type proximity).

1. $C_{H}$ remains an equilibrium allocation of competition in contracts if $p_{L}$ is decreased (distance between types is increased).

2. $C_{H}$ becomes an equilibrium allocation of mechanism competition if $p_{L}$ is sufficiently small (distance between types is sufficiently increased).

Maskin and Tirole (1992) showed that allowing the principal to offer practically unrestricted mechanisms (or game forms), rather than contracts in the narrow sense, eliminates a certain type of inefficiency. Indeed, in simple models, signalling costs are sometimes higher than strictly necessary, simply because the agent's off-equilibrium beliefs force the principal to pay these costs. By offering a "menu" in which incentive constraints are binding, the principal himself can eliminate these costs in advance.

This effect is limited here. Certain equilibria we find with price competition and contract competition disappear when menus are allowed, but not all. Indeed, when types are very dissimilar, it remains better not to deviate from profitable offers to $H$ : menus that are attractive to the consumer must offer something attractive to an $L$ (else it suffices that the consumer believes that he is an $L$ when the menu is offered to see him refuse the menu), but as the offer becomes more attractive to $L$, it necessarily becomes less profitable on $H$. This discourages deviations.

\subsection{More Than Two Types}

Consider competition in contracts with a "large" type support. We prove that, for any type, the set of allocations he can receive increases when the support of the prior distribution increases. Retrospectively, $H$ and $L$ in the two-type study can be considered as a particular pair of the more general distribution.

We consider a finite support of types denoted by $\mathcal{K}$ in which $J$ and $K$ are generic types. Let $\lambda_{J}$ and $\lambda_{K}$, the prior probabilities of $J$ and $K$, be strictly positive. The two-type "conditional game" where the support of the types is restricted to $J$ and $K$, and where the proportions of the types are, 
respectively $\frac{\lambda_{J}}{\lambda_{J}+\lambda_{K}}$ and $\frac{\lambda_{K}}{\lambda_{J}+\lambda_{K}}$ exhibits exactly the characteristics we studied in previous sections. We denote the set of equilibrium strategy profiles of this game by $\mathcal{E}(J, K)$. A generic element of this set is denoted by $\left(C_{J}^{\bullet}, C_{K}^{\bullet}\right)$, where, e.g., $C_{J}^{i}$ would be insurer $i$ 's offer if a $J$ enters the market.

Proposition 4 Partition $\mathcal{K}$ in pairs and singletons, and denote by $\left(J_{s}, K_{s}\right)$ the $s^{\text {th }}$ "pair" (a singleton if $J_{s}=K_{s}$ ), with $s \in S, S$ being a minimal set of indices. For each $s \in S$, select an equilibrium strategy profile of the conditional game $\left(C_{J_{s}}^{\bullet}, C_{K_{s}}^{\bullet}\right) \in \mathcal{E}\left(J_{s}, K_{s}\right)$.

We have the following result: either $\left(C_{J_{s}}^{\bullet}, C_{K_{s}}^{\bullet}\right)_{s \in S}$ itself, or an arbitrarily close approximation, is an equilibrium strategy profile of the full-support game.

Remark that $\left(C_{J_{s}}^{\bullet}, C_{K_{s}}^{\bullet}\right)_{s \in S}$ is not always an equilibrium since two different pairs could lead to the same contracts, in which case the restrictions on the beliefs inherited from the two conditional games are conflicting. The proposition states that in that case, we can always find another equilibrium for one of the conditional games such that the ambiguity disappears, the strong result being that this other equilibrium can be chosen as close to the original equilibrium as we may wish.

There are other types of equilibria, e.g. where three or more types are pooled. Pooling allocations need not group types that are close in the support: in a four-type economy, extreme types can form one pooling and intermediate ones another.

The result points to an essential mechanism behind multi-sender games: they entail a high degree of coordination on the public signal (the consumer's type). In consequence, individual insurers taken in isolation have little influence in general, and often none, on the allocation: they are virtually forced to align their offer with the others', even if certain deviations could be extremely valuable.

\subsection{Refinements}

Refinements are conceived for eliminating equilibria which are supported by somehow "unreasonable" beliefs. After examining the impact of eliminating discontinuous beliefs, we test existing refinements. We simply recall that refinements are either focussed on the principals' incentives, or directly concerned with the revelation of information. Readers interested must read Hertzendorf and Overgaard (2001) for a complete discussion and contributions on this topic. 
Continuous Beliefs ${ }^{17}$ It is often useful, for the sake of exposition, to show that inefficiencies are preserved because potential deviations are discouraged by discrete effects on the beliefs. For example, a strictly profitable $C_{H}$ in a separating equilibrium could be maintained because a better offer $C^{\prime}$ causes optimistic beliefs that makes even $C^{\prime}$ unacceptable. The question is therefore: are inefficiencies maintained only because of discontinuous beliefs? The answer, we prove, is no.

To define continuous beliefs, we take the Euclidian measure on $\Omega_{1}^{N}$ (a space of dimension $2 N$ - two states, $N$ insurers-).

Proposition 5 If beliefs $\widetilde{p}(\cdot)$ are continuous in a symmetric equilibrium (pooling or separating), then any equilibrium offer is either (1) actuarially fair or (2) just acceptable.

In summary, continuous beliefs select extreme allocations in which either insurers or the consumer do not gain from trade.

The second category being more unexpected, we still have to check that the Bertrand logic can be broken in effect and that the equilibrium offer can be just acceptable and unfair. Take a symmetric allocation in which $C_{H}$ is unfair, and equivalent to $C^{0}$ for $\widetilde{p}=p_{H}$ (condition 2 in the proposition). For a unilateral deviation $C \neq C^{0}$, define $p(C)$ as the belief for which $C$ is equivalent to no insurance. Define $\widetilde{p}(\cdot)$ such that $\widetilde{p}\left(C, C_{H}, \ldots, C_{H}\right)<p(C)$ when $p(C)>p_{L}$ and $\widetilde{p}\left(C, C_{H}, \ldots, C_{H}\right) \geq p_{L}$. By construction, a deviation by insurer 1 is not acceptable when it is profitable and not profitable when it is acceptable (the same restriction must be imposed for any other insurer's deviation), and $\widetilde{p}(\cdot)$ can be chosen to be continuous. ${ }^{18}$ Pooling equilibria are sustained by similar procedures.

Incentive Based Refinements In these refinements, one starts from the equilibrium allocation and tries to see what types of principal would "gain" from playing a deviation. Beliefs associated should put zero mass on the types that do not gain. Different versions of this refinement vary as to what "gain" exactly means, namely, what sort of reaction from the part of the uninformed agent should be expected to compute the payoff consistently (this has been

\footnotetext{
${ }^{17}$ We thank a referee for suggesting that we examine this question.

${ }^{18}$ Our reasoning is still valid if we take Lipschitzian beliefs (which is more demanding than mere continuity), i.e. we can assume that there is a positive number $M$ such that

$$
\left|\widetilde{p}\left(C^{\bullet}\right)-\widetilde{p}\left(C^{\bullet \prime}\right)\right| \leq M \cdot\left\|C^{\bullet}-C^{\bullet \prime}\right\|
$$

since $p(C)$ is continuous and Lipschitzian, which implies that $\widetilde{p}(\cdot)$ can be Lipschitzian as well.
} 
essentially developed in the principal-agent context). The example that we will develop is the Intuitive Criterion of Cho and Kreps (1987), notoriously efficient in many signalling games.

We apply the classical definition ${ }^{19}$ as follows: in all equilibria, we verify that the contribution to beliefs of any insurer is "reasonable". Otherwise stated, if $C_{H}^{\bullet *}$ and $C_{L}^{\bullet *}$ are equilibrium vectors of offers, beliefs $\tilde{p}$ are reasonable if and only if for any $i$ and for any $K$, beliefs $\tilde{p}\left(C_{K}^{-i *}, \cdot\right)$ are reasonable as far as insurer $i$ 's behavior is concerned.

Proposition 6 A PBE allocation is robust to the Cho-Kreps criterion if and only if (1) it is separating and (2) it assigns the actuarially fair full insurance contract to the low-risk type.

A certain degree of efficiency is reached via the refinement. Non-discriminating equilibria are eliminated, as well as inefficient insurance for the low risk in separating equilibria. However, both strictly profitable insurance and noinsurance for $H$ are robust.

The failure can be understood as follows. Even if the high risk is not insured whereas the low risk is completely insured at an actuarially fair price, deviations that are favorable to the high-risk consumer (contracts better than no-insurance) are typically favorable to the insurer whatever the agent's real type.

Revelation Based Refinements This category is specific to multi-principal environments. The idea is that in a given equilibrium, vectors of offers for different types are typically different in more than one dimension. The consequence is that no principal's offer is essential for interpreting correctly the message: any short list of offers (even one offer only) is sufficiently informative in most equilibria. Each insurer is tempted to free-ride the revelation part, and attract the consumer with the best deal. However, notice that the reasoning is valid ex post only, i.e. too late in a pure PBE.

Bagwell and Ramey (1991) proposed that equilibrium beliefs should be

\footnotetext{
${ }^{19}$ Take a PBE. Given a type $K$, some propositions of the insurer would lead it to earn, whatever the subsequent beliefs of the agent provided he acts conditionally on them, a profit which is lower (strictly lower for one possible belief at least) than the profit it earns by playing its equilibrium action. The intuitive criterion imposes the following restriction: a "reasonable" belief of the agent associated with such a dominated proposition must put zero mass on type $K$, provided that the proposition gives a higher profit than equilibrium profit on the remaining type. If the condition is not satisfied, the equilibrium is eliminated.
} 
unprejudiced in the following sense. ${ }^{20,21}$ To each vector of offers, they associate two numbers: the first (resp. the second) is the number of offers different from the equilibrium offers assigned to the high-risk (resp. the low-risk) type. If these two numbers differ, the belief associated with the examined pair should put probability one on the type corresponding to the strictly smaller number. Applied to our model, their refinement selects as a unique separating equilibrium actuarially fair full insurance for each type. Indeed, individual insurers do not have influence on beliefs when they deviate, which means that they can easily propose better contracts without seeing customers flee. However, the criterion is incapable of eliminating pooling equilibria.

Combining Refinements The intuitive criterion is useful against pooling equilibria whereas the Bagwell-Ramey criterion is useful against inefficient separating equilibria. There is another difference. With the intuitive criterion, the dimension of the contract space is crucial for the arguments based on incentives. Applied to price competition, the criterion is incapable of eliminating pooling equilibria. With unprejudiced beliefs, the dimension of the contract space is irrelevant.

Applying both arguments sequentially (the order doesn't matter) leads to the unique allocation that the market would have reached under symmetric information: actuarially fair full insurance for each type. We must however bear in mind that the implicit informational and cognitive assumptions are largely obscure, which is a common (and valid) objection against refinements.

\subsection{Public Offers}

Our analysis is focused on individual markets. In markets where people with similar characteristics form large identifiable groups, insurers could post target public offers. Pooling equilibria, we have shown, are necessarily profitable on both types. Now suppose that types are independently distributed in the group. If, through a public offer, an insurer commits to sell insurance at slightly better conditions to anyone from the group, then people should not update their beliefs and should accept this offer. This would be mutually advantageous for the deviating insurer and consumers. ${ }^{22}$

In fact, there is no unique way to introduce groups and public offers in the model nor a unique way to adapt Bayesian revision rules. For example,

\footnotetext{
${ }^{20}$ The economic problem treated in their model (oligopoly pricing when the quality of the product is not observed by the buyer) is very different from ours (no common value in their model).

${ }^{21}$ Hertzendorf and Overgaard (2001) propose a wider discussion on the topic.

${ }^{22}$ We thank a referee for raising this issue.
} 
types could be correlated in the group (there is no compelling reason why draws by Nature should be independent), to the point that all members are either $H$ or $L$. (Public causal factors like criminality rates, jurisprudence, climatic evolution, etc., are ready examples where correlation is likely.) In that case, the individual analysis recovers its strength: the public offer can still be interpreted by the group as a signal that they are better than average, and they all prefer to remain uninsured.

These two extreme situations aim at proving that the analysis of group offers requires several additional assumptions on the statistical relationship between the members, and on the kind of sophistication we want to put in their beliefs. A full clarification of the issue is left for further research.

\subsection{Mandatory Insurance}

The analysis provides a rationale for mandatory insurance. Consider a pooling equilibrium. An offer that is slightly better for all beliefs always exists (it suffices to increase the indemnity by a small amount), and will necessarily be preferred, to the profit of the deviating insurer. Taken to its logical conclusion, this eliminates all pooling in equilibrium, because no insurer would accept losses on high risk consumers. Profits are therefore necessarily zero, and allocations are separating and actuarially fair. However, the improvement is not complete since overinsurance for $L$ or underinsurance for $H$ is possible. Denote by $C_{L}^{H}$ the optimal insurance contract for an $H$ that is actuarially fair for an $L$ (this contract offers overinsurance to $L$ ). We show now that $C_{L}^{H}$ is an equilibrium allocation for $L$. Indeed, if when one insurer at least offers another fair contract than $C_{L}^{H}$ then $\widetilde{p}=p_{H}$, then the equilibrium is necessarily $C_{L}^{H}$. This proves that the optimal coverage is not warranted by mandatory insurance.

\section{Conclusion}

Competition on individual markets does not ensure perfect revelation of information to consumers, and even when market interactions reveal information, the allocation may remain inefficient. The irony is that the largest rents to the firms are left by the most distrustful consumers, those who refuse to admit they have the costly type, until they finish - too late - with an inefficient lot. As refinements indicate, sophisticated consumers are better treated by the market: educating the public's understanding of insurance, not to say of competition, is likely to produce desirable effects. The efficacy of educationbased policies is even stronger when risks change faster than regulations. 
Regulating discrimination in insurance markets is particularly difficult. Ex ante, all equilibria of our game are worse than the allocation that would emerge under symmetric ignorance of the types (namely, average fair full insurance). We encounter a variety of the Hirshleifer paradox, where the value of the insurers' superior information is socially negative. ${ }^{23}$ The tragedy is that better rating techniques are always sought because they enable the insurer to attract selectively, and profitably, the lower risk consumers. Even if, sooner or later, imitation undermines the informational advantage, search for better rating threatens the status quo, whatever it is. Moreover, if antidiscrimination laws could break this dynamic, the danger would come from the other side: adverse selection (better informed consumers) can suddenly become an issue, and the very laws that protect the consumer against useless discrimination become an obstacle to efficiency.

The easiest tests of our theory are based on predictions which do not contradict our model but which falsify that of Rothschild and Stiglitz, and the symmetric information hypothesis. In the absence of a quantitative argument, we suggest that the following statements are reasonably attractive: (1) low risk people may be better covered by insurance than high risk people; (2) certain people are not insured in spite of their above average riskiness; (3) in certain niches, policyholders are persistently profitable. ${ }^{24}$

Notice that our statements are not ceteris paribus. We base our analysis on the fact that two people may be observationally different for the insurers and the econometrician, and still not be aware of the extent of their differences in riskiness. Compared to the tests of adverse selection, this imposes a different treatment of control variables. In theory, the most complete view of insurance markets lies between extremes: both parties have private information and menus signal information from insurers to individuals whereas individuals reveal information to insurers by their choices. Whether the structure of asymmetric information is close to one of the extremes (adverse selection or better informed insurers) is an empirically challenging question. Given that in practice, insurers offer menus in which consumers make their

\footnotetext{
${ }^{23}$ Pooling equilibria in our model are less favorable to the individual since they entail positive profits on both types. In the separating equilibria, even the best case where each type is fully insured according to its actuarial value, the individual receives ex ante less utility than with the uninformative, average fair, full insurance. Indeed, the uninformed risk-averse consumer does not want to incur the risk of paying a price that depends on his type, especially because the average price remains the same.

${ }^{24}$ These consequences differ from "proselection" (better risks are better covered) as defined in Hemenway (1990), since one suspects that individuals there are better informed. He showed that careless people (those who do not fasten their seat belts) tend to have adopted less insurance coverage. The data were collected at a car rental company by direct observation.
} 
choices, but menus are not the same for all individuals (see Puelz and Snow, 1994, and Chiappori and Salanié, 2000), econometricians have to control for the exact nature of offers and classification techniques in order not to assess asymmetric information where there is none.

\section{A References}

Bagwell, K., Ramey, G., 1991. Oligopoly Limit Pricing. RAND Journal of Economics 22, 155-172.

Chiappori, P.A., Salanié, B., 2000. Testing for Asymmetric Information in Insurance Markets. Journal of Political Economy 108, 56-78.

Cho, I.K., Kreps, D.M., 1987. Signaling Games and Stable Equilibria. Quarterly Journal of Economics 102, 179-221.

Cohen, A., 2001. Asymmetric Information and Learning in the Automobile Insurance Market. Mimeo, Harvard University. 
Rothschild, M., Stiglitz, J.E., 1976. Equilibrium in Competitive Insurance Markets : An Essay on the Economics of Imperfect Information. Quarterly Journal of Economics 90, 629-649.

Stiglitz, J.E., 1977. Monopoly, Non-Linear Pricing and Imperfect Information : The Insurance Market. Review of Economic Studies 44, 493-510.

Villeneuve, B., 2000. The Consequences for a Monopolistic Insurance Firm of Evaluating Risk Better than Customers: The Adverse Selection Hypothesis Reversed. The Geneva Papers on Risk and Insurance Theory $25,65-79$.

Wolinsky, A., 1995. Competition in Markets for Credence Goods. Journal of Institutional and Theoretical Economics 151,117-131.

\section{B Proofs}

\section{B.1 Proposition 1}

Let $c_{H}^{\bullet}$ and $c_{L}^{\bullet}$ be the vectors of offers to, respectively, type- $H$ and type- $L$ consumers. 1. and 2. follow the same logic: there are contracts that are profitable and acceptable whatever the subsequent beliefs of the consumer. These contracts lie in $\left(c_{L}^{\mathrm{AF}}, c_{L}^{\max }\right)$ for $L$, in $\left(c_{H}^{\mathrm{AF}}, c_{L}^{\max }\right)$ for $H$. Competition is severe, and still, two offers at least should and do charge the minimal price (else, the only insurer offering this contract would have some leeway to raise its price). 3. Here the logic is inverted: a contract that is both profitable and acceptable for all the possible subsequent beliefs of the consumer doesn't exist. In other words, if we associate any profitable deviation with a belief such that the deviant offer is not acceptable (which is always possible by taking, e.g., $\left.p_{L}\right)$ and given that the beliefs associated to non-profitable deviations need not be restricted, then deviations are discouraged.

\section{B.2 Proposition 2}

Conditions (a) and (b) are stated in the text above the Proposition.

1. Remark that $c_{A}^{\max } \rightarrow c_{H}^{\max }$ monotonically as $\lambda_{H} \rightarrow 1$ : increasing $\lambda_{H}$ increases the set of acceptable contracts for an uninformed agent. This relaxes (b), strictly when the set of pooling is not empty, weakly when it is. 2 . N only affects the RHS in (a) and (b); if $p_{L}>0$, then (b) becomes violated for a large $N$. 3. For $p_{L}=0$, (b) is always satisfied. The proportion of $L$ must be low enough $\left(c_{A}^{\max }\right.$ close enough to $\left.c_{H}^{\max }\right)$ for (a) to be met. By continuity, 
and given that the constraints on pooling equilibria are slack at $p_{L}=0$, small positive values of $p_{L}$ and $\lambda_{L}$ guarantee existence.

\section{B.3 Proposition 3}

We prove that if all insurers offer $C_{H}$ to $H$ (offering a single contract is a mechanism), then we can find beliefs such that no mechanism is an attractive deviation. We show that this is possible if $p_{L}$ is sufficiently small.

For all $\mu \in \Omega_{2}$, all $K, K(\mu)$ denotes the allocation that $\mu$ assigns to $K$. We distinguish two cases (a) $L(\mu)$ makes a profit strictly higher than $\pi_{L}^{\max }$ on $L$ if accepted, and (b) $L(\mu)$ makes a profit lower than $\pi_{L}^{\max }$ on $L$, if accepted. We check now that the following beliefs sustain the equilibrium allocation (no-insurance for all): if (a) then consumer believes he is an $L$; if (b) then he believes he is an $H$.

(a) $\mu$ would break the equilibrium only if $L(\mu)$ were strictly preferable to no-insurance for belief $p_{L}$. This is impossible.

(b) $\mu$ breaks the equilibrium only if (i) $H(\mu)$ is strictly preferable to no-insurance for belief $p_{H}$ (the value of $\mu$ for the individual is the value of $H(\mu)$ ), (ii) $H(\mu)$ is strictly profitable on $H$ (the insurer has to be induced to deviate) and (iii) more profitable than $L(\mu)$ on $H$ (incentive compatibility). Without loss of generality, $K(\mu)$ can be seen as a random allocation of insurance contracts: the mechanism works as if $K(\mu)$ were specifying the random rules according to which $\left(W_{N}, W_{A}\right)$ were allocated. Let us define $\tilde{\mu}$ as the insurance (ordinary) contract $\left.\left(E\left(W_{N} \mid H(\mu)\right), E\left(W_{A} \mid H(\mu)\right)\right)\right)$. Due to the insurer's risk neutrality and the consumer's risk aversion, if $\mu$ breaks the equilibrium, then (i) $\tilde{\mu}$ is also strictly preferable to no-insurance for belief $p_{H}$, (ii) $\tilde{\mu}$ is also strictly profitable on $H$, and (iii) still more profitable than $L(\mu)$ on $H$. Therefore $\tilde{\mu}$ (hence $H(\mu)$ ) necessarily generates strictly positive profits on $L$. On the other hand, incentive compatibility ensures that $L(\mu)$ is more profitable than $H(\mu)$ on $L$, otherwise stated, profits on $H(\mu)$ are necessarily bounded above by a certain $\pi_{H}\left(p_{L}\right)$ that tends towards zero as $p_{L}$ tends towards zero. This means that the deviation becomes necessarily unattractive as $p_{L}$ gets smaller, and the equilibrium is not broken.

\section{B.4 Proposition 4}

Base case. Consider the following three pairs: (a) $C_{J_{s}}^{\bullet}$ and $C_{J_{s^{\prime}}}^{\bullet}$, (b) $C_{K_{s}}^{\bullet}$ and $C_{K_{s^{\prime}}}^{\bullet}$, and (c) $C_{J_{s^{\prime}}}^{\bullet}$ and $C_{K_{s}}^{\bullet}$. If, for all $s, s^{\prime}$, the above pairs of vectors have at least two different contracts, then $\left(C_{J_{s}}^{\bullet}, C_{K_{s}}\right)_{s \in S}$ is exactly supported in an equilibrium. To see this, notice that in each of the conditional games, beliefs are restricted only for equilibrium offers and unilateral deviations. Beliefs 
associated with vectors of offers differing in two or more dimensions from one of the equilibrium vectors are not restricted at all. The restricted beliefs of the conditional games can be transplanted in the complete game without interference, and they sustain the allocation.

Other cases. Assume that one of the conditions above is violated, e.g. $C_{J_{s}}^{\bullet}$ and $C_{J_{s^{\prime}}}^{\bullet}$, without loss of generality, differ in less than two dimensions. We show that $C_{J_{s}}^{\bullet}$ and $C_{J_{s^{\prime}}}^{\bullet}$ can be replaced by arbitrarily close equilibrium allocations so as to eliminate ambiguity and go back to the base case. We deal with two subcases: (a) $\left(C_{J_{s}}^{\bullet}, C_{K_{s}}^{\bullet}\right)$ or $\left(C_{J_{s^{\prime}}}^{\bullet}, C_{K_{s^{\prime}}}^{\bullet}\right)$ is a pooling equilibrium; (b) $\left(C_{J_{s}}^{\bullet}, C_{K_{s}}^{\bullet}\right)$ and $\left(C_{J_{s^{\prime}}}^{\bullet}, C_{K_{s^{\prime}}}^{\bullet}\right)$ are separating.

(a) To fix ideas, assume that $\left(C_{J_{s}}^{\bullet}, C_{K_{s}}^{\bullet}\right)$ is a pooling equilibrium. Notice that there is a continuum of pooling equilibria in $\mathcal{E}\left(J_{s}, K_{s}\right)$. This means that we can choose $\varepsilon \in \mathbb{R}^{2}$ with $\|\varepsilon\|>0$ as small as necessary), such that, if we add vector $\varepsilon$ to the conditional transfers $\left(W_{N}, W_{A}\right)$ implicit in $C_{J_{s}}^{\bullet}$ and $C_{K_{s}}^{\bullet}$ to eliminate any ambiguity caused by the resemblance with $C_{J_{s^{\prime}}}^{\bullet}$ and $C_{K_{s^{\prime}}}^{\bullet}$, the modified offers still support a pooling equilibrium. We are back to the base case.

(b) If $J_{s}$ and $J_{s^{\prime}}$ were both low risks in their conditional games, they would receive actuarially fair offers in separating allocations. But $J_{s} \neq J_{s^{\prime}}$, therefore their vectors of offers would be sufficiently different. This proves that one type, say $J_{s}$ to fix ideas, is the high risk in "his" conditional game. The set of equilibrium allocations for $J$ being a continuum, ${ }^{25}$ the offers he obtains can be slightly changed to approximate $C_{J_{s}}^{\bullet}$ and eliminate ambiguity. We are back to the base case.

\section{B.5 Proposition 6}

(1) Take a symmetric pooling equilibrium. We denote the proposed contract by $C^{*}$, and by $\pi_{H}^{*}$ and $\pi_{L}^{*}$ the associated profits $\left(\pi_{K}^{*}=\pi_{K}\left(C^{*}\right) / N\right)$. Define $C$ as the barycenter of $C^{0}$ (weight $N-1$ ) and $C$ (weight 1); we have $\pi_{K}(C)=\pi_{K}^{*} / N$ for any $K$. The VNM utility functions being quasi-concave, $C$ is necessarily strictly acceptable for belief $p_{H}\left(C^{0}\right.$ and $C^{*}$ are acceptable for belief $p_{A}$ ), and strictly better than $C^{*}$. Following the CK logic, in the neighborhood of $C$, the angular sector in which $\pi_{H}(\cdot)>\pi_{H}^{*} / N$ and $\pi_{L}(\cdot)<\pi_{L}^{*} / N$ is such that $\tilde{p}\left(C^{*}(N-1\right.$ times $\left.), \cdot\right)=p_{H}$. An individual insurer would offer a contract sufficiently close to $C$ in this sector: the consumer is attracted, and profits are superior. The equilibrium is broken.

(2) Fix $i$ and consider $\tilde{p}\left(C_{L}^{-i *}, \cdot\right)$. The refinement imposes that for any $C^{i}$

\footnotetext{
${ }^{25}$ This is obvious with price competition, and it remains true in general because sustainable allocations are defined by regular incentive constraints.
} 
generating losses on $H$ and profits on $L$, in particular around $C_{L}^{\mathrm{AF}}, \tilde{p}\left(C_{L}^{-i *}, C^{i}\right)$ must be $p_{L}$. If the type- $L$ consumer receives an actuarially fair but inefficient allocation, then there must necessarily be a deviation by $i$ in the neighborhood of $C_{L}^{\mathrm{AF}}$ that is acceptable, better than the equilibrium offer, and strictly profitable. This eliminates unfair or inefficient allocations to $L$. We cannot go further. Consider $\tilde{p}\left(C_{H}^{-i *}, \cdot\right)$. Whatever $C^{i}$ offered by $i$ that would improve its profit on $H, C^{i}$ would not decrease its equilibrium profit on $L$ (which is zero). In consequence, beliefs cannot be restricted by the Cho-Kreps criterion and the equilibrium is not eliminated. 


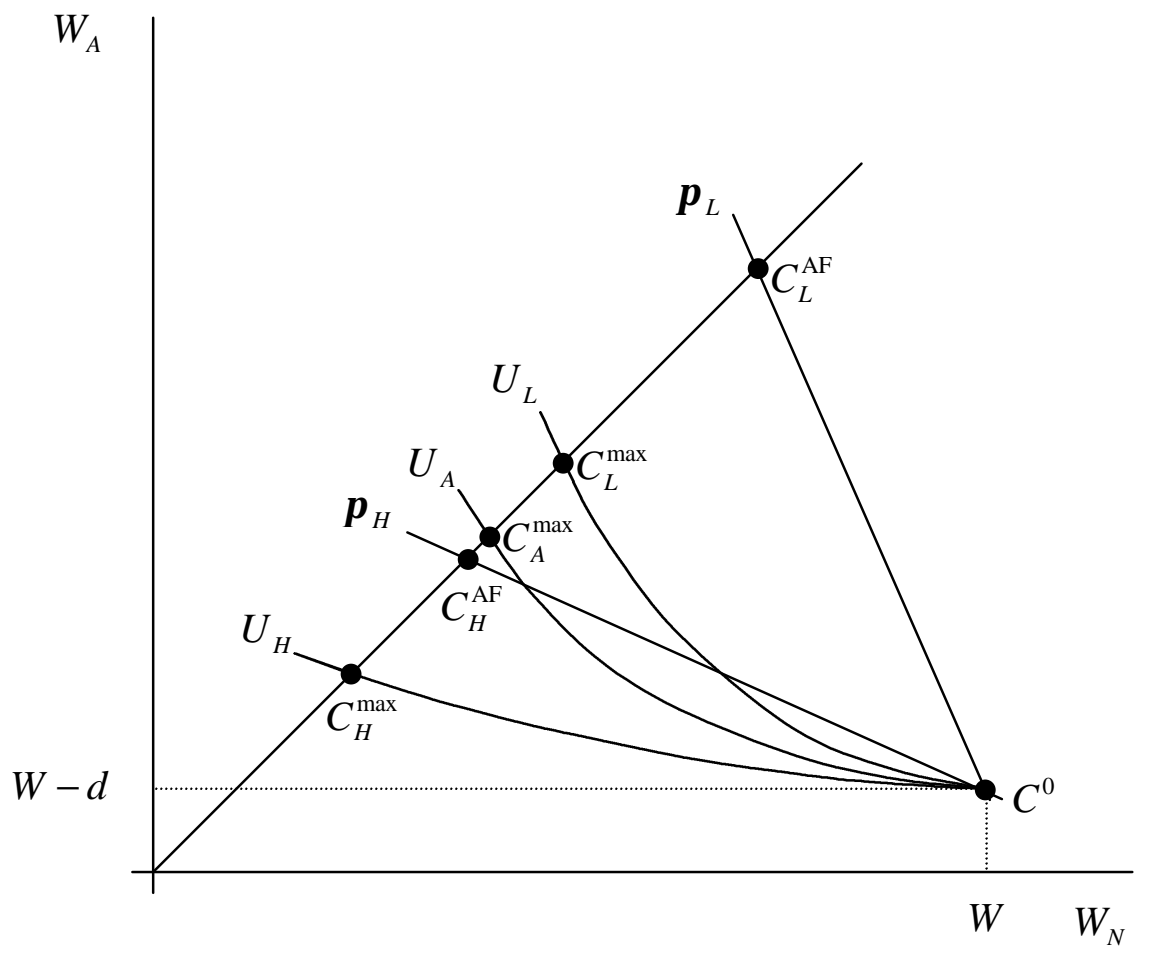

Figure 1 


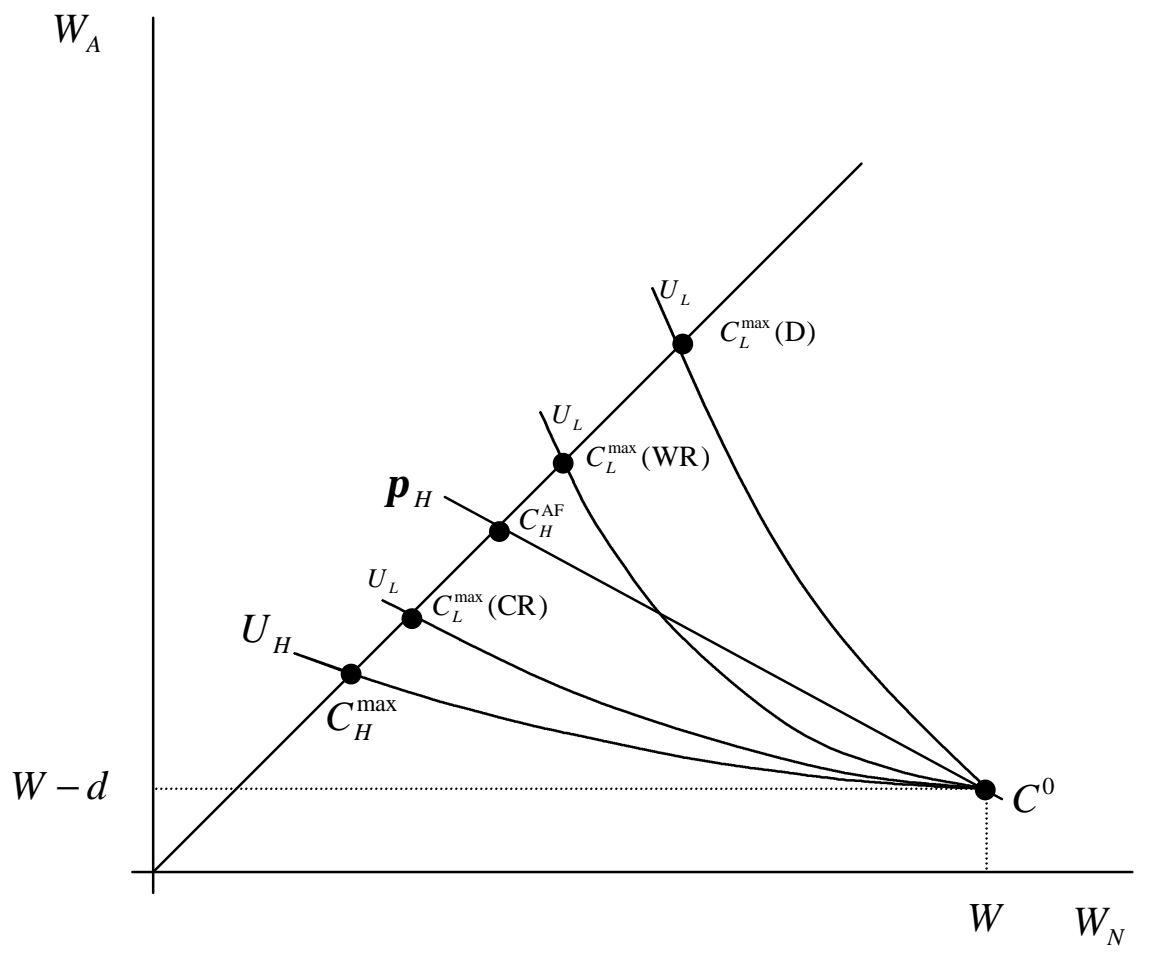

Figure 2 